\title{
Validation d'une technique de dosage multiélémentaire des métaux et métalloïdes dans les cheveux par ICP-MS. Valeurs de référence chez 45 témoins
}

\section{ICP-MS multi-elementary metal and metalloid validation in hair : reference values in 45 healty volunteers}

\section{Jean-Pierre GOULLÉ ${ }^{(1) *}$, Loïc MAHIEU(1), Laurent BONNEAU ${ }^{(1)}$, Gilbert LAINE(1), Daniel BOUIGE ${ }^{(2)}$, Christian LACROIX ${ }^{(1)}$}

(1) Laboratoire de Pharmacocinétique et de Toxicologie Cliniques, Groupe Hospitalier du Havre BP 24 - 76083 LE HAVRE CEDEX

(2) Laboratoire de Biochimie, Groupe Hospitalier du Havre - BP 24 - 76083 LE HAVRE CEDEX

*Auteur à qui adresser la correspondance : Jean-Pierre GOULLÉ, Laboratoire de Pharmacocinétique et de Toxicologie Cliniques, Groupe Hospitalier du Havre - BP 24 - 76083 LE HAVRE CEDEX - France Tél : 0232733223 - Fax : 0232733238 -E-mail : jgoulle@ch-havre.fr

Ce travail a été présenté au XII Congrès annuel de la SFTA à Porticcio

(Reçu le 24 mars 2005 ; accepté après modifications le 31 mai 2005)

\section{RÉSUMÉ}

Nous décrivons une technique de dosage simultané de trente deux éléments minéraux dans les cheveux, par plasma à couplage inductif relié à un détecteur de masse (ICP-MS). L'appareil utilisé est un spectromètre X7CCT Thermo Elemental, sans cellule dynamique de réaction. Quarante cinq volontaires des deux sexes, indemnes de toute affection et sans traitement médical constituent les témoins. Une mèche de cheveux est prélevée au niveau du vertex postérieur: Après décontamination de $25 \mathrm{mg}$ de cheveux, puis minéralisation acide, trente deux métaux et métalloïdes sont

\begin{abstract}
SUMMARY
A thirty-two metal and metalloid quantification method for hair using inductively coupled plasma mass spectrometry (ICP-MS), is presented. The ICP-MS measurements were performed using a Thermo Elemental X7CCT series and Plasma Lab ${ }^{\circledR}$ software without a dynamic reaction cell. Posterior vertex hair from forty-five healthy volunteers, of both sexes, were collected. Twenty-five mg hairs were acid mineralized after a decontanination procedure, and thirtytwo metals and metalloids were simultaneously quantified after acid dilution following water calibration. $L i, B e, B, A l$,
\end{abstract}


quantifiés simultanément par dilution de la solution acide obtenue. L'étalonnage est réalisé en milieu aqueux. Les dosages sont validés pour les métaux suivants: $L i, B e, B, A l$, V, Cr, Mn, Co, $\mathrm{Ni}, \mathrm{Cu}, \mathrm{Zn}, \mathrm{Ga}, \mathrm{Ge}, \mathrm{As}, \mathrm{Se}, \mathrm{Rb}, \mathrm{Sr}, \mathrm{Mo}, \mathrm{Pd}, \mathrm{Ag}$, $\mathrm{Cd}, \mathrm{Sn}, \mathrm{Sb}, \mathrm{Te}, \mathrm{Ba}, \mathrm{W}, \mathrm{Pt}, \mathrm{Hg}, \mathrm{Tl}, \mathrm{Pb}, \mathrm{Bi}, \mathrm{U}$. Les coefficients de régression sont supérieurs à 0,999 dans la ganme de linéarité considérée. Les linnites de quantification s'échelonnent de $0,2 \mathrm{pg} / \mathrm{mg}(\mathrm{Tl}, \mathrm{Pt}, U)$ à $0,5 \mathrm{ng} / \mathrm{mg}(B)$. Les valeurs obtenues sont présentées et discutées à la lumière des rares données disponibles. Les progrès récents de l'ICP-MS constituent un apport déterminant en biologie clinique et plus particulièrement dans le domaine de l'analyse toxicologique en raison de la puissance de l'outil. Son application à l'analyse multiélémentaire des métaux dans les cheveux offre des perspectives nouvelles tant pour la recherche d'intoxications en toxicologie clinique ou judiciaire que pour la surveillance d'expositions professionnelles ou environnementales.

\section{MOTS-CLÉS}

ICP-MS, métaux, métallö̈des, cheveux.

\section{Introduction}

Dans un précédent numéro, nous avons décrit et validé une technique de dosage multiélémentaire par torche à plasma (ICP-MS) dans le sang total, le plasma, les urines (1). Plus récemment nous avons déterminé les valeurs usuelles dans ces milieux pour cent témoins (2). Il nous a semblé intéressant d'appliquer cette technique aux cheveux. Rappelons que le dosage des métaux ou métallö̈des dans cette matrice constitue l'application la plus ancienne du dosage des xénobiotiques dans les cheveux. Au $19^{\text {ent }}$ siècle, l'intoxication arsenicale aiguë est relativement courante, et celle-ci est mortelle dans bon nombre de cas. C'est ainsi que dès 1836, Marsh met au point une technique de dosage de l'arsenic dans les cheveux. La sensibilité de la méthode permet de déceler $0,5 \mu \mathrm{g}$ d'anhydride arsénieux. Pour l'époque, si c'est une prouesse technique exceptionnelle, la constatation de l'accumulation de l'arsenic dans les phanères de sujets régulièrement exposés est tout aussi capitale. Elle ouvre un nouveau champ d'investigation dans l'analyse toxicologique: l'analyse des xénobiotiques dans les cheveux, domaine qui a connu un essor particulièrement important depuis 15 ans. Dans les cheveux, si l'analyse de métalloïdes que sont l'arsenic ou le thallium, ou bien d'un métal comme le mercure ont des indications bien établies, de nombreuses controverses sont nées quant à l'interprétation des mesures tant pour les oligo-éléments que pour de nombreux métaux. Parmi ces controverses, outre une mauvaise fixation de la plupart de ces éléments dans la matrice protéique, l'exposition en milieu industriel pouvant être à l'origine d'une contamination externe des cheveux est parfois difficile à éliminer avant analyse.
V, Cr, Mn, Co, Ni, Cu, Zn, Ga, Ge, As, Se, Rb, Sr; Mo, Pd, Ag, $\mathrm{Cd}, \mathrm{Sn}, \mathrm{Sb}, \mathrm{Te}, \mathrm{Ba}, \mathrm{W}, \mathrm{Pt}, \mathrm{Hg}, \mathrm{Tl}, \mathrm{Pb}, \mathrm{Bi}, \mathrm{U}$ hair determination could be validated. Linearity was excellent and the correlation coefficients were above 0.999. Quantification limits ranged from $0.2 \mathrm{pg} / \mathrm{mg}$ (Te, Pt, $U$ ) to $0.5 \mathrm{ng} / \mathrm{mg}(B)$. Results are presented and compared to rare published data. ICP-MS has made significant advances in the field of clinical biology, particularly in toxicological analyses. This is due to the use of extremely effective equipment that contributes to improved clinical, forensic, toxicological diagnosis which in turn permits greater control of industrial or environmental exposure.

\author{
KEY-WORDS \\ ICP-MS, metals, metalloids, hair:
}

\section{Matériel et méthodes}

Il s'agit d'une torche à plasma de type ThermoElectron $\mathrm{X}$ Series couplée à un spectromètre de masse, modèle X7/CCT (ThermoElectron, Courtaboeuf, France).

L'appareil est équipé d'une torche en quartz de $1,5 \mathrm{~mm}$, d'un nébuliseur concentrique en verre borosilicaté de 1 $\mathrm{mL}$ (type concentrique de marque Glass ExpansionRéférence ThermoElemental 1201318) avec un débit d'échantillon de $0,85 \mathrm{~mL} / \mathrm{min}$, d'une chambre de nébulisation en quartz munie d'un refroidisseur à effet Peltier régulant sa température à $3^{\circ} \mathrm{C}$, et d'un passeur d'échantillons de type CETAC ASX-510. L'ensemble des données est enregistré sur une station informatique dotée du logiciel d'analyse PlasmaLab version 2.0 sous Windows NT. Les paramètres instrumentaux sont les suivants : puissance de la torche $1200 \mathrm{~W}$; débits d'argon : plasmagène $15 \mathrm{~L} / \mathrm{min}$, nébuliseur $0,95 \mathrm{~L} / \mathrm{min}$, auxiliaire $0,66 \mathrm{~L} / \mathrm{min}$; interface : cônes échantillonneurs et écorceurs en nickel de diamètre respectifs de 1 $\mathrm{mm}$ et de $0,4 \mathrm{~mm}$; vide au niveau de l'interface : 1,9 mbar et du quadripôle : 1,6.10-7 mbar. Les réactifs, de qualité suprapur pour analyses de traces et les solutions étalon de métaux proviennent de chez Merck (Darmstadt, Allemagne) et CPI (Amsterdam, Hollande). Après décontamination par de l'acétone et de l'eau tiède, $25 \mathrm{mg}$ de cheveux sont minéralisés par $250 \mu \mathrm{l}$ d'acide nitrique suprapur à $70^{\circ} \mathrm{C}$ pendant une heure (soit $10 \mathrm{mg}$ pour $100 \mu \mathrm{l}$ ). La solution est ensuite refroidie une heure à $-20^{\circ} \mathrm{C}$. A $100 \mu \mathrm{l}$ de la solution acide obtenue (il est donc possible de réduire la prise d'essai à $10 \mathrm{mg})$, on ajoute $3900 \mu \mathrm{l}$ de diluant $(0,5 \%$ de butanol, $1 \%$ d'acide nitrique, In et $\mathrm{Rh} 1 \mathrm{ppb}, 0,01$ $\%$ triton). Trente deux éléments sont quantifiés simultanément dans les cheveux : lithium, béryllium, bore, 
aluminium, vanadium, chrome, manganèse, cobalt, nickel, cuivre, zinc, gallium, germanium, arsenic, sélénium, rubidium, strontium, molybdène, palladium, argent, cadmium, étain antimoine, tellure, baryum, tungstène, mercure, thallium, plomb, bismuth, uranium. L'étalonnage est réalisé en milieu aqueux. Le protocole de validation est celui recommandé par la Société Française de Biologie Clinique (3). L'exactitude des mesures est contrôlée par l'emploi de cheveux titrés pour 23 éléments auprès du contrôle de qualité Canadien. Trois fois par an le laboratoire participe au programme de comparaison inter-laboratoires organisé par l'institut national de santé publique du Québec (Sainte Foy - Canada). Dans les deux cas, il s'agit de cheveux dopés pour la majorité des métaux ainsi que pour l'arsenic.

\section{Résultats}

Les paramètres de la procédure de validation : élément ainsi que l'isotope quantifié, linéarité, limite de détection (LD), limite de quantification (LQ), répétabilité et reproductibilité sont regroupés dans le tableau I. Pour

Tableau I : Linéarité, limite de détection $(L D)$, limite de quantification $(L Q)$, répétabilité et reproductibilité.

\begin{tabular}{|c|c|c|c|c|c|}
\hline $\begin{array}{l}\text { Élément } \\
\text { isotope }\end{array}$ & $\begin{array}{c}\text { Iinéarité } \\
\mathrm{r}\end{array}$ & $\begin{array}{l}\text { Limite de détection } \\
\text { en ng/mg }\end{array}$ & $\begin{array}{l}\text { Limite de quantification } \\
\text { en ng/mg }\end{array}$ & $\begin{array}{l}\text { Répétabilité } \\
\text { CV \% }\end{array}$ & $\begin{array}{l}\text { Reproductibilité } \\
\text { CV \% }\end{array}$ \\
\hline $7 \mathrm{Li}$ & 0,9999 & 0,002 & 0,007 & 6,5 & 6,1 \\
\hline 9Be & 0,9998 & 0,002 & 0,007 & 3,9 & 8,8 \\
\hline $11 \mathrm{~B}$ & 0,9991 & 0,14 & 0,46 & 3,6 & 8,9 \\
\hline $27 \mathrm{AI}$ & 0,9993 & 0,02 & 0,08 & 2,3 & 7,7 \\
\hline $51 \mathrm{~V}$ & 0,9998 & 0,001 & 0,003 & 1,7 & 9,0 \\
\hline $53 \mathrm{Cr}$ & 0,9999 & 0,06 & 0,20 & 3,5 & 9,3 \\
\hline $55 \mathrm{Mn}$ & 0,9996 & 0,001 & 0,004 & 1,7 & 6,6 \\
\hline $59 \mathrm{Co}$ & 0,9998 & 0,0003 & 0,001 & 2,3 & 7,9 \\
\hline $60 \mathrm{Ni}$ & 0,9998 & 0,01 & 0,05 & 1,8 & 6,4 \\
\hline $65 \mathrm{Cu}$ & 0,9999 & 0,01 & 0,03 & 1,3 & 10,4 \\
\hline $66 \mathrm{Zn}$ & 0,9996 & 0,01 & 0,04 & 1,1 & 8,1 \\
\hline 69Ga & 0,9998 & 0,0003 & 0,0009 & 2,2 & 8,9 \\
\hline $74 \mathrm{Ge}$ & 0,9999 & 0,001 & 0,002 & 1,8 & 7,6 \\
\hline $75 \mathrm{As}$ & 0,9997 & 0,01 & 0,02 & 3,5 & 6,4 \\
\hline $82 \mathrm{Se}$ & 0,9997 & 0,02 & 0,06 & 2,6 & 7,8 \\
\hline $85 \mathrm{Rb}$ & 0,9995 & 0,0003 & 0,001 & 2,0 & 5,8 \\
\hline $88 \mathrm{Sr}$ & 0,9995 & 0,0002 & 0,0007 & 1,0 & 7,0 \\
\hline $98 \mathrm{Mo}$ & 0,9998 & 0,0004 & 0,001 & 3,9 & 8,2 \\
\hline 105Pd & 0,9995 & 0,001 & 0,003 & 2,9 & 22,3 \\
\hline $107 \mathrm{Ag}$ & 0,9998 & 0,0005 & 0,002 & 0,7 & 9,9 \\
\hline $111 \mathrm{Cd}$ & 0,9998 & 0,0003 & 0,0009 & 0,7 & 5,9 \\
\hline $118 \mathrm{Sn}$ & 0,9998 & 0,001 & 0,002 & 1,0 & 5,9 \\
\hline $121 \mathrm{Sb}$ & 0,9998 & 0,0003 & 0,001 & 1,0 & 5,2 \\
\hline $125 \mathrm{Te}$ & 0,9997 & 0,0006 & 0,002 & 6,7 & 6,1 \\
\hline 137Ba & 0,9998 & 0,001 & 0,003 & 0,8 & 5,5 \\
\hline $182 W$ & 0,9998 & 0,0002 & 0,001 & 2,1 & 7,2 \\
\hline $195 \mathrm{Pt}$ & 0,9999 & 0,0001 & 0,0002 & 1,5 & 6,2 \\
\hline $202 \mathrm{Hg}$ & 0,9986 & 0,004 & 0,013 & 0,4 & 9,5 \\
\hline 203TI & 0,9995 & 0,00005 & 0,0002 & 3,7 & 4,7 \\
\hline $208 \mathrm{~Pb}$ & 0,9997 & 0,0003 & 0,001 & 0,7 & 4,4 \\
\hline $209 \mathrm{Bi}$ & 0,9997 & 0,0008 & 0,003 & 1,4 & 5,3 \\
\hline $238 \mathrm{U}$ & 0,9998 & 0,00004 & 0,0002 & 2,0 & 7,2 \\
\hline
\end{tabular}


les 32 éléments étudiés, la droite de la régression linéaire reliant les concentrations et les signaux mesurés est supérieure à 0,999 . Les limites de détection et de quantification obtenues sont pour les éléments les plus sensibles inférieures au $\mathrm{pg} / \mathrm{mg}$. Ainsi les limites de quantification s'échelonnent de $0,2 \mathrm{pg} / \mathrm{mg}$ soit $0,2 \mathrm{ng} / \mathrm{g}$ pour le thallium, le platine et l'uranium à $0,5 \mathrm{ng} / \mathrm{mg}$ pour le bore. En ce qui concerne la répétabilité celle-ci est toujours inférieure à $3,9 \%$ à l'exception du lithium $(6,5 \%)$ et du tellure $(6,7 \%)$. La reproductibilité est toujours inférieure à $10 \%$ à l'exception du palladium $(22,3 \%)$. Les résultats obtenus au contrôle de qualité canadien pour les 23 paramètres disponibles sont reportés dans le tableau II. Outre la valeur mesurée, le tableau indique, la valeur cible (médiane) calculée à partir des concentrations obtenues par les participants ainsi que le «Z score». Celui-ci est établi après une troncature préalable à deux écarts-types. L'analyse statistique permet ensuite de calculer la médiane et l'écart type pour chaque élément. Les critères d'acceptabilité

Tableau II : Résultats obtenus au contrôle de qualité canadien (ICP04 H-03).

\begin{tabular}{|c|c|c|c|}
\hline Élément & $\begin{array}{c}\text { Valeur Mesurée } \\
\text { (ng/mg) }\end{array}$ & $\begin{array}{c}\text { Médiane } \\
\text { (ng/mg) }\end{array}$ & $\begin{array}{c}\mathbf{Z} \text { score } \\
|\mathbf{Z}|<2\end{array}$ \\
\hline $\mathbf{A g}$ & 0,44 & 0,44 & 0,02 \\
\hline $\mathbf{A l}$ & 33 & 57 & $-6,23$ \\
\hline $\mathbf{A s}$ & 0,4 & 0,3 & 0,98 \\
\hline $\mathbf{B a}$ & 10,3 & 10 & 0,37 \\
\hline $\mathbf{B e}$ & 0,19 & 0,17 & 0,39 \\
\hline $\mathbf{C d}$ & 0,68 & 0,67 & 0,08 \\
\hline $\mathbf{C o}$ & 0,23 & 0,25 & $-0,24$ \\
\hline $\mathbf{C r}$ & 1 & 1,1 & $-0,65$ \\
\hline $\mathbf{C u}$ & 25,4 & 28 & $-1,15$ \\
\hline $\mathbf{H g}$ & 0,22 & 0,23 & $-0,08$ \\
\hline $\mathbf{M n}$ & 3,1 & 3,4 & $-0,87$ \\
\hline $\mathbf{M o}$ & 0,21 & 0,24 & $-0,33$ \\
\hline $\mathbf{N i}$ & 1,7 & 1,4 & 1,22 \\
\hline $\mathbf{P b}$ & 12,1 & 11 & 1,13 \\
\hline $\mathbf{P t}$ & 0,52 & 0,33 & 1,72 \\
\hline $\mathbf{S b}$ & 0,65 & 0,6 & 0,4 \\
\hline $\mathbf{S e}$ & 3,4 & 3,8 & $-1,16$ \\
\hline $\mathbf{S n}$ & 1,1 & 1,3 & $-0,94$ \\
\hline $\mathbf{T e}$ & 0,5 & 0,39 & 0,89 \\
\hline $\mathbf{T l}$ & 0,18 & 0,17 & 0,08 \\
\hline $\mathbf{U}$ & 0,10 & 0,10 & $-0,03$ \\
\hline $\mathbf{V}$ & 0,24 & 0,31 & $-0,70$ \\
\hline $\mathbf{Z n}$ & 164 & 180 & $-1,40$ \\
\hline
\end{tabular}

sont indiqués dans le tableau MII. La qualité des résultats est appréciée par le calcul de Z-score qui s'effectue de la manière suivante :

$$
\mathrm{Z}=\frac{\text { résultat rendu }- \text { valeur cible }}{\text { écart attribué }}
$$

En l'absence de valeur cible connue, on utilise pour le calcul de Z-score, la concentration médiane calculée après troncature. Si la valeur absolue de $\mathrm{Z}$ est inférieure à 2 , les résultats sont considérés comme bons; si cette valeur est supérieure à 3 , ceux-ci ne sont pas satisfaisants. Hormis l'aluminium pour lequel la valeur obtenue du $\mathrm{Z}$ score est supérieure à 2 , les résultats que nous avons obtenus sont bons. Les valeurs de référence sont établies chez 45 témoins des deux sexes (tableau IV). Pour chaque élément, le pourcentage de résultats înférieurs à la limite de détection, la médiane, la dispersion des mesures et les valeurs normales sont indiqués.

Tableau III : Critères d'acceptabilité pour le calcul du Zscore.

\begin{tabular}{|c|c|c|}
\hline Concentration ng/mg & $\begin{array}{c}\text { Erreur maximale } \\
\text { acceptable }\end{array}$ & Eeart attribué \\
\hline$<0,5$ & $100 \%$ & $33,3 \%$ \\
\hline $0,5-2$ & $50 \%$ & $16,7 \%$ \\
\hline $2-5$ & $30 \%$ & $10 \%$ \\
\hline$>5$ & $20 \%$ & $6,7 \%$ \\
\hline
\end{tabular}

\section{Discussion}

En ce qui concerne la validation du dosage des 32 éléments minéraux dans les cheveux, les performances obtenues sont excellentes pour 31 métaux et métalloïdes. La droite de la régression linéaire reliant les concentrations aux signaux mesurés est bonne puisque $\mathrm{R}^{2}$ est toujours au moins égal à 0,9991 (B). Les limites de détection (LD) et de quantification calculées (LQ) sont particulièrement faibles. Ainsi pour le platine, le thallium, l'uranium, la LD s'établit respectivement à $0,1-0,05-0,04 \mathrm{pg} / \mathrm{mg}$ avec une LQ identique pour ces trois éléments $(0,2 \mathrm{pg} / \mathrm{mg})$. Ces valeurs sont comparables à celles obtenues avec la chromatographie liquide couplée à la spectrométrie de masse tandem (LCMS-MS) pour certains xénobiotiques commé le bromazépam dans les cheveux, médicament pour lequel la LQ est de $0,2 \mathrm{pg} / \mathrm{mg}$ (4). Les performances obtenues avec l'ICP-MS sont d'autant plus remarquables qu'elles correspondent à une prise d'essai de $10 \mathrm{mg}$ de cheveux. Pour le palladium, la médiocre reproductibilité obtenue (coefficient de variation à $22,3 \%$ ) a vraisemblablement pour origine l'emploi dans le même local d'un 
Tableau IV : Valeurs de référence chez 45 témoins des deux sexes.

\begin{tabular}{|c|c|c|c|}
\hline & \% < à LD & $\begin{array}{c}\text { Médiane } \\
\text { ng/mg }\end{array}$ & $\begin{array}{c}\text { Dispersión } \\
\mathbf{5}^{\mathbf{9}} \mathbf{\text { percentile }} \\
\text { ng/mg }\end{array}$ \\
\hline Lithium & 0 & 0,016 & $0,003-0,042$ \\
\hline Bérylium & 0 & 0,007 & $0,003-0,012$ \\
\hline Bore & 0 & 0,54 & $0,26-1,87$ \\
\hline Aluminium & 4 & 1,63 & $0,26-5,30$ \\
\hline Vanadium & 20 & 0,016 & $0,001-0,051$ \\
\hline Chrome & 0 & 0,20 & $0,11-0,52$ \\
\hline Manganèse & 4 & 0,067 & $0,016-0,570$ \\
\hline Cobalt & 0 & 0,023 & $0,004-0,14$ \\
\hline Nickel & 0 & 0,23 & $0,08-0,90$ \\
\hline Cuivre & 0 & 20,3 & $9,0-61,3$ \\
\hline Zinc & 0 & 162 & $129-209$ \\
\hline Gallium & 0 & 0,011 & $0,002-0,068$ \\
\hline Germanium & 7 & 0,004 & $<0,001-0,039$ \\
\hline Arsenic & 0 & 0,05 & $0,03-0,08$ \\
\hline Sélénium & 0 & 0,54 & $0,37-1,37$ \\
\hline Rubidium & 0 & 0,006 & $0,003-0,03$ \\
\hline Strontium & 0 & 0,89 & $0,17-4,63$ \\
\hline Molybdène & 2 & 0,021 & $0,010-0,028$ \\
\hline Palladium & 0 & 0,010 & $0,004-0,049$ \\
\hline Argent & 0 & 0,08 & $0,02-1,31$ \\
\hline Cadmium & 0 & 0,011 & $0,004-0,17$ \\
\hline Étain & 0 & 0,046 & $0,007-0,34$ \\
\hline Antimoine & 0 & 0,008 & $0,003-0,13$ \\
\hline Tellure & 89 & 0,0003 & $0,0003-0,001$ \\
\hline Baryum & 0 & 0,28 & $0,05-1,58$ \\
\hline Tungstène & 9 & 0,0013 & $0,0001-0,007$ \\
\hline Platine & 87 & 0,00035 & $0,00035-0,0008$ \\
\hline Mercure & 0 & 0,66 & $0,31-1,66$ \\
\hline Thallium & 0 & 0,0002 & $0,0001-0,0004$ \\
\hline Plomb & 0 & 0,41 & $0,13-4,57$ \\
\hline Bismuth & 18 & 0,009 & $0,0004-0,14$ \\
\hline Uranium & 0 & 0,009 & $0,002-0,03$ \\
\hline & & & \\
\hline
\end{tabular}

sel de palladium comme modificateur de matrice pour réaliser des dosages d'aluminium par spectrophotométrie d'absorption atomique électrothermique. Le dosage du palladium dans les cheveux peut toutefois être utilisé dans un contexte d'exposition significative. En ce qui concerne le chrome, contrairement aux autres matrices biologiques (sang total, plasma, urines) où le recours à une cellule de collision s'avère indispensable, en raison d'interférences spectrales, la validation de ce paramètre dans les cheveux n'a posé aucun problème, avec de bonnes performances au contrôle d'assurance de qualité organisé par l'institut national de santé publique du québec. Excepté pour l'aluminium, les résultats obtenus à ce contrôle de qualité sont bons, très bons ou excellents. Hormis l'argent, le cuivre, le mercure et le sélénium qui sont présents à des concentrations normales, les autres éléments ont été fortifiés à des concentrations 1,5 fois à 17 fois les valeurs supérieures normales, et respectivement à 200 fois et 500 fois celles-ci pour le tellure et pour le platine. Les valeurs de référence établies chez nos 45 témoins appellent un certain nombre de commentaires. Si elles sont très souvent inférieures aux concentrations publiées par ICP-AES ; nous constatons en revanche que nos résultats sont très voisins de ceux obtenus sur 1091 sujets par Miekeley, par ICP-MS (5). Ainsi pour les 20 éléments communs à ces deux études, nous trouvons pour 8 d'entre eux des normales sensiblement supérieures, pour 10 autres des normales sensiblement inférieures et pour 2 paramètres des normales identiques. En ce qui concerne les applications de l'analyse des métaux et métalloïdes dans les cheveux, de nombreux travaux ont été publiés (6-13). Ils concernent des analyses réalisées dans le cadre d'intoxications ou d'expositions à certains métaux comme le mercure, l'arsenic, le plomb ou le cadmium. Le déficit ou l'excès d'éléments essentiels connus comme le calcium, le zinc, le sélénium, le cuivre et le chrome ont également été étudiés. Selon les publications, les résultats obtenus conduisent à des analyses parfois très divergentes. Cela tient également au fait qu'il est plus difficile pour le cheveu que pour d'autres matrices biologiques d'établir des valeurs normales, en particulier pour les éléments présents en faible concentration et pour les éléments trace. En effet, des variations importantes peuvent être constatées, selon les éléments, en fonction du sexe, de l'âge, de l'origine ethnique et géographique du sujet, de la couleur de ses cheveux, de ses habitudes alimentaires. D'autre part, au cours des dernières années, les diverses équipes ont essentiellement utilisé l'ICP-AES, technique nettement moins sensible que l'ICP-MS (tableau IV), conduisant régulièrement de ce fait à des valeurs usuelles plus élevées en raison de limites de détection supérieures par la technique optique comparée à la spectrométrie de masse. Enfin, depuis 5 ans des progrès importants sont constatés dans les limites de détections par spectrométrie de masse comme le montre le tableau IV. Malgré l'amélioration de la sensibilité, celle-ci est encore insuffisante pour le tellure chez $89 \%$ des témoins, pour le platine chez $87 \%$ de la population étudiée, pour le vanadium et le bismuth respectivement chez $20 \%$ et $18 \%$ des sujets. Si l'on com- 
Tableau V: Sensibilités comparées de l'ICP-AES et de l'ICP-MS..

\begin{tabular}{|c|c|c|c|c|c|c|}
\hline & \multicolumn{2}{|c|}{ ICP - AES (Miekeley) } & \multicolumn{2}{c|}{ ICP-MS (Miekeley) } & \multicolumn{2}{c|}{ ICP-MS (ce travail) } \\
\hline & Longueur d'onde & LD & Isotope & LD $(3 \mathbf{E T}$ & LD & LQ \\
\hline $\mathbf{A g}$ & 328,068 & 0,02 & 107 & 0,001 & 0,0005 & 0,002 \\
\hline $\mathbf{A s}$ & 189,042 & 0,2 & 75 & 0,04 & 0,01 & 0,02 \\
\hline $\mathbf{A u}$ & 242,795 & 0,4 & 197 & 0,001 & - & - \\
\hline $\mathbf{B i}$ & 223,061 & 0,8 & 209 & 0,001 & 0,0008 & 0,003 \\
\hline $\mathbf{C d}$ & 228,802 & 0,4 & 111 & 0,006 & 0,0003 & 0,0009 \\
\hline $\mathbf{C} \mathbf{0}$ & 228,616 & 0,12 & 59 & 0,001 & 0,0003 & 0,001 \\
\hline $\mathbf{C r}$ & 267,716 & 0,14 & 53 & 0,01 & 0,06 & 0,20 \\
\hline $\mathbf{G e}$ & 209,426 & 0,8 & 73 & 0,01 & 0,001 & 0,002 \\
\hline $\mathbf{H g}$ & 194,232 & 0,8 & 202 & 0,005 & 0,004 & 0,013 \\
\hline $\mathbf{M} \mathbf{N i}$ & 202,030 & 0,16 & 98 & 0,003 & 0,0004 & 0,001 \\
\hline $\mathbf{N i}$ & 231,604 & 0,3 & 62 & 0,01 & 0,01 & 0,05 \\
\hline $\mathbf{P b}$ & 220,353 & 1,8 & $206-208$ & 0,003 & 0,0003 & 0,001 \\
\hline $\mathbf{S b}$ & 206,833 & 1,2 & 121 & 0,005 & 0,0003 & 0,001 \\
\hline $\mathbf{S e}$ & 196,090 & 1,5 & 82 & 0,05 & 0,02 & 0,06 \\
\hline $\mathbf{S n}$ & 189,989 & 1,6 & 118 & 0,03 & 0,001 & 0,002 \\
\hline $\mathbf{T h}$ & 283,730 & 0,4 & 232 & 0,001 & - & - \\
\hline $\mathbf{U}$ & 385,958 & 0,6 & 238 & 0,001 & 0,00004 & 0,0002 \\
\hline
\end{tabular}

Les résultats sont exprimés en $\mathrm{ng} / \mathrm{mg}$ ou $\mu \mathrm{g} / \mathrm{g}$

$\mathrm{ET}=$ écart type $-\mathrm{LD}=$ limite de détection $-\mathrm{LQ}=$ limite de quantification

pare les valeurs normales obtenues chez nos 45 témoins à des cohortes plus importantes, en particulier celle de Rio forte de 1091 sujets, à l'exception du bore, nous obtenons des résultats sensiblement équivalents. Ainsi nous trouvons des normes un peu plus élevées pour 8 paramètres (vanadium, chrome, cobalt, nickel, cuivre, argent, antimoine, bismuth), un peu plus basses pour 10 paramètres (aluminium, manganèse, zinc, germanium, arsenic, molybdène, cadmium, baryum, mercure, plomb) et strictement identiques pour le strontium et l'étain. Ces écarts peuvent s'expliquer pour les raisons diverses évoquées plus haut. Les modalités différentes de lavage sont aussi une source de variation possible. Le dosage multiélémentaire des métaux et métalloïdes dans les cheveux par ICP-MS avec double secteur magnétique a été proposé (14-16). Cette’technique permet la détermination sinultanée de 71 éléments avec des performances analytiques excellentes. Enfin, plus récemment la spéciation de l'arsenic dans les cheveux a été mise au point dans le cadre d'une des plus grandes intoxications collectives de l'histoire au Bengladesh $(17,18)$.

\section{Conclusion}

L'ICP-MS est une technique spécifique et d'une grande sensibilité. Elle permet de quantifier à partir d'un échantillon réduit de cheveux, une trentaine de métaux et métalloïdes simultanément dans un délai relativement court. La méthode décrite est particulièrement adaptée à des dosages en série. Elle offre des perspectives nouvelles non seulement en toxicologie clinique et médico-légale, mais également dans les domaines de la pathologie professionnelle et de la surveillance environnementale.

Cet équipement a été acquis grâce à un financement de l'Association pour la Fondation Charles Nicolle Rouen - France (Président Professeur Eric MALLET). 


\section{Références}

1. Goullé J.P., Mahieu L., Castermant J., Neveu N., Lainé G., Nouveau M.P., Gehanne R., Bouige D., Lacroix C. Validation d'une technique de dosage multiélémentaire des métaux par ICP-MS dans les milieux biologiques. Ann. Toxicol. Anal. $2003 ; 15$ : 271-80. Correctif dans Ann. Toxicol. Anal. $2004 ; 16$ : 257-60.

2. Goullé J.P., Mahieu L., Castermant J., Neveu N., Lainé G., Nouveau M.P., Gehanne R., Bouige D., Lacroix C. Dosage multiélémentaire des métaux et métallö̈des dans les milieux biologiques par ICP-MS : valeurs usuelles chez 100 témoins. Ann. Toxicol. Anal. 2004 ; 16 : 261-268.

3. Protocole de validation de techniques. Commission validation de techniques de la Société Française de Biologie Clinique. L'information Scientifique du Biologiste. 1985 ; $11: 5-51$.

4. Villain M., Cheze M., Dumestre V., Ludes B., Kintz P. Hair document drug-facilitated crimes : four cases involving bromazépam. J. Anal. Toxicol. 2004 ; 28 : 516-9.

5. Miekeley N., Dias Carneiro M.T.W., Porto da Silveira C.L. How reliable are human hair reference intervals for trace elements? Sci. Total Environ. 1998 ; 218 : 9-17.

6. Tong S.L., Zhu W.Z., Gao Z.H., Meng Y. X., Peng R.L., Lu G.C. Distribution characteristics of rare earth elements in children's scalp hair from a rare earths mining area in southern China. J. Environ. Sci. Health. 2004 ; 39 : 2517 32.

7. Hasan M.Y., Kosanovic M., Fahim M.A., Adem A., Petroianu G. Trace metal profiles in hair samples from children in urban and rural regions of the United Arab Emirates. Vet. Hum. Toxicol. 2004 ; 46 : 119-121.

8. Bencko V. Use of human hair as a biomarker in the assessment of exposure to pollutants in occupational and environmental settings. Toxicology $1995 ; 101: 29-39$.

9. Porto da Silveira C.L., Miekeley N., de Carvalho Fortes L.M., de Paula F.A. Alkaline earth element and phosphorus anomalies in human hair sample as an indicator for osteoporosis. Fifth COMTOX Symposium on Toxicology and Clinical Chemistry of Metals. Vancouver, Canada. Abstract $1995 ; 311: 78$.
10. Miekeley N., de Fortes Cavahlo L.M., Porto da Silveira C.L., Lima M.B. Elemental anomalies in hair as indicators of endocrinologic pathologies and deficiencies in calcium and bone metabolism. J. Trace Elem. Med. Biol. 2001 ; $15: 46-55$.

11. Razagui I. B., Haswell S. J. The determination of mercury and selenium in maternal and neonatal scalp hair by inductively coupled plasma-mass spectrometry. J. Anal. Toxicol. $1997 ; 21: 149-53$.

12. Samanta G., Sharma R., Roychowdhury T., Chakraborti D. Arsenic and other elements in hair, nails, and skin-scales of arsenic victims in West Bengal, India. Sci. Total Environ. 2004 ; 326 : 33-47.

13. Marquet P., Francois B., Lotfi H., Turcant A., Debord J., Nedelec G., Lachatre G. Tungsten determination in biological fluids, hair and nails by plasma emission spectrometry in a case of severe acute intoxication in man. J. Forensic Sci. 1997 ; 42 : 527-30.

14. Rodushkin I., Axelsson M.D. Application of double focusing sector field ICP-MS for multielemental characterization of human hair and nails. Part I. Analytical methodology. Sci. Total Environ. $2000 ; 250: 83-100$.

15. Rodushkin I., Axelsson M.D. Application of double focusing sector field ICP-MS for multielemental characterization of human hair and nails. Part II. A study of the inhabitants of northern Sweden. Sci. Total Environ. 2003 ; $262: 21-36$.

16. Rodushkin I., Axelsson M.D. Application of double focusing sector field ICP-MS for multielemental characterization of human hair and nails. Part III. Direct analysis by laser ablation. Sci. Total Environ. 2003 ; 305 : 23-39.

17. Mandal B.K., Ogra Y., Suzuki K.T. Speciation of arsenic in human nail and hair from arsenic-affected area by HPLC-inductively coupled argon plasma mass spectrometry. Toxicol. Appl. Pharmacol. 2003 ; 189 : 73-83.

18. Mandal B.K., Ogra Y., Suzuki K.T. Speciation of arsenic in biological samples. Toxicol. Appl. Pharmacol. 2004 ; 198 : 307-18. 